\section{Toxicological evaluation of chronic oral administration of Melissa officinalis hydro-ethanol extract in Sprague-Dawley rats}

\author{
Mohammad Hashemnia, ${ }^{1}$ Farid Rezaei, ${ }^{1}$ \\ Zahra Nikousefat,2 Maral Bahiraei ${ }^{1}$ \\ 1Department of Pathobiology, \\ Veterinary Medicine Faculty, Razi \\ University, Kermanshah; ${ }^{2}$ Department \\ of Clinical Sciences, Veterinary \\ Medicine Faculty, Razi University, \\ Kermanshah, Iran
}

\section{Abstract \\ Melissa officinalis is a plant that} has been widely used as an herbal medicine in many countries. Unfortunately, despite the prevalent medicinal uses of the plant, there are no reports on the possible toxic effects of $M$. officinalis. This study was designed to evaluate the effect of long-term administration of hydro-alcoholic extract of M. officinalis on some biochemical and hematological parameters and histopathology of organs. Thirty Sprague-Dawley rats were allocated to three equal groups. The animals in groups A and B received 600 and $1200 \mathrm{mg} / \mathrm{kg} \mathrm{M}$. officinalis extract, respectively, for 30 days. The rats in group $\mathrm{C}$ were given gavaged saline as control. The animals were euthanized at the end of experiment and the blood samples were collected for biochemical and hematology analysis. Additionally, appropriate tissue samples were collected from kidney, liver, spleen, heart and lung for light microscopic examination. $M$. officinalis caused a significant increase in the alanine aminotransferase level in the treated rats. Although the increase in creatine phosphokinase and lactate dehydrogenase levels were observed in group A and B, respectively, but there were no significant differences. A significant decrease was observed in the total protein and albumin concentrations in serum of treated rats as compared to the control group. The creatinine concentrations were significantly higher in the group B when compared to the other groups. There were no significant differences in cholesterol, triglyceride and urea concentrations between all groups of rats. The main histopathologic findings in the liver were included hepatocyte degeneration, congestion and dilation of sinusoids, proliferation of bile ducts and infiltration of mononuclear cells around the portal area. Histopathologic examination of the kidneys showed a tubular degeneration and necrosis, tubular and glomerular atrophy and congestion. These lesions were more prominent in the high dose treated rats. The findings suggest that long-term administration of $M$. officinalis extract even at low doses induces hepatic and renal lesions in rats.

\section{Introduction}

Medicinal plants are popular remedies used by a majority of the world's population. The efficacy of medicinal plant in the management of diseases is unquestionable. The World Health Organization estimated that $80 \%$ of the people in different countries use plants as their primary source of medication. ${ }^{1}$ Plants commonly used in traditional medicine are assumed to be safe and their toxicity and pharmacological aspects are not considered. This safety is based on their long usage in the treatment of diseases according to knowledge accumulated over centuries. Recent evidence suggests that some of the herbs considered to be safe over the last many decades have proven to be associated with health hazards. Adverse reactions may result from irrational usage, such as excess dosage. Furthermore, medicinal plants can act either as agonists or antagonists that potentiate some drug therapies. ${ }^{2}$ Information on the toxicity of the plants is very important as a baseline before further exploring its development as a new herbal medicine. ${ }^{3}$ Balm (Badrangboya in Persian) (Melissa officinalis L.), member of the family Lamiaceae (formerly Labiatae) is an aromatic (lemony) perennial herb, up to about $1 \mathrm{~m}$ high, growing in the Mediterranean region, western Asia, southwestern Siberia, and northern Africa. ${ }^{4}$

Lemon balm is a medicinal plant as well as most important commercial plants during the recent decades. ${ }^{5}$ In folk medicine, balm has been traditionally used for different medical purposes as tonic, antispasmodic, carminative, diaphoretic, surgical dressing for wounds and relief of stress induced headache. ${ }^{6}$ Additionally, it is very useful for promoting sleep, nervous complaints, lower abdominal disorders, gastric complaints, migraine, hysteria and melancholia, nervous debility, toothache, earache and high blood pressure. 4

Balm contains volatile oil, glycosides of the alcoholic or phenolic components of the volatile oil (eugenol glucoside), caffeic acid derivatives (rosmarinic acid), flavonoids (cynaroside, cosmosin, rhamnocitrin, isoquercitrin), phenolic acid (carnosic acid), and triterpene acids (ursolic and oleanolic
Correspondence: Mohammad Hashemnia, Department of Pathobiology, School of Veterinary Medicine, Razi University, P.O. Box: 67156-85414, Kermanshah, Iran. Tel.: +98.831.8322599 / 8329540

E-mail:m.hashemnia@razi.ac.ir

Key words: Melissa officinalis, Histopathological and biochemical parameters, Toxicity, Rat.

Acknowledgments: We thank the authorities of Veterinary School, Razi University for their cooperation.

Contributions: the authors contributed equally.

Conflict of interest: the authors declare no potential conflict of interest.

Received for publication: 8 November 2015 Revision received: 14 December 2015. Accepted for publication: 8 January 2016.

This work is licensed under a Creative Commons Attribution NonCommercial 4.0 License (CC BY-NC 4.0).

CCopyright M. Hashemnia et al., 2017 Licensee PAGEPress srl, Italy

Veterinary Science Development 2017; 7:6298 doi:10.4081/vsd.2017.6298

acid). The last two compounds are well known antioxidants. ${ }^{7}$ Despite widespread use of $M$. officinalis as herbal medicine, no toxicological data is available regarding the safety of its long-term consumption. Therefore, as part of a safety evaluation of M. officinalis, a toxicological study was carried out to investigate its potential toxicity after 30-day repeated oral dosing of $M$. officinalis ethanolic extract in rats.

\section{Materials and Methods}

\section{Animals}

Adult Sprague-Dawley rats of both sexes weighing 200 to 220 g were purchased from University of Medical Sciences (Kermanshah, Iran). The animals were housed under standard environmental conditions $\left(23 \pm 1{ }^{\circ} \mathrm{C}\right.$, with $55 \pm 5 \%$ humidity and a $12 \mathrm{~h} \mathrm{light/dark} \mathrm{cycle)} \mathrm{and} \mathrm{maintained}$ with free access to water and ad libitum standard laboratory diet (70\% carbohydrates, $25 \%$ proteins, $5 \%$ lipids). The rats were randomly assigned into three diabetic and one control group $(n=10)$. The study was approved by the local ethics committee of our faculty, in accordance with the ethics standards of Principles of Laboratory Animal Care. 


\section{Plant material and hydro-alcoholic extract preparation}

Fresh leaves of $M$. officinalis was obtained from the local market. Plant specimen was subjected to air-drying for three days and then the dried leaves was grounded into powder. The powder was extracted by $1 \mathrm{~L}$ of hydro-ethanol mixture $(80 / 20$, $\mathrm{v} / \mathrm{v})$ for $8 \mathrm{~h}$. This step was repeated for four times. The filtrate was pooled and concentrated under vacuum at a temperature, not exceeding $60^{\circ} \mathrm{C}$. The alcoholic extract was stored at $-20^{\circ} \mathrm{C}$ until being used.

\section{Experimental design}

In the present experiment, 30 rats were used. The rats were divided into three groups. Ten rats were used in each group. The animals in groups A and B received 600 and $1200 \mathrm{mg} / \mathrm{kg}$ body weight $M$. officinalis extract, respectively. The rats in group $\mathrm{C}$ were given gavage saline as control.

At the end of the experiment, animals from each group were weighed, euthanized and blood and tissues were obtained for biochemical, hematological and histopathological analysis.

An automated cell counter (Sysmex K1000) was used to analyze blood for hematological parameters, to determine the hemoglobin concentration $(\mathrm{Hb})$, red blood cell count (RBC), packed cell volume (PCV), Mean corpuscular volume (MCV) and mean corpuscular hemoglobin concentration (MCHC). For serum, clotted blood was centrifuged for $10 \mathrm{~min}$ at $3000 \mathrm{rpm}$ and separated sera stored at $-20^{\circ} \mathrm{C}$ until analyzed. Serum samples were analyzed by Hitachi autoanalyzer for the activities of aspartate aminotransferase (AST), alanine aminotransferase (ALT) and concentrations of lactate dehydrogenase (LDH), creatine phosphokinase (CPK), albumin, cholesterol, triglyceride, total protein, creatinine and urea. For histopathological evaluation, appropriate tissue samples were collected from the kidney, liver, spleen, heart and lung were then fixed in $10 \%$ neutral buffered formalin, embedded in paraffin, sectioned at $5 \mu \mathrm{m}$ thickness, and stained with hematoxylin-eosin staining for light microscopic examination. Description and scoring of histopathological lesions were performed according to the method of Ozden et al. (2005). ${ }^{8}$

\section{Statistical analysis}

For hematological and biochemical parameters analysis, descriptive statistics including the mean, standard deviation, median, minimum and maximum were calculated for all variables. The ANOVA and Tukey tests were used for comparison of different parameters. For histopathological evaluation, differences among groups were evaluated using X2 and Kruskal-Wallis tests. Data were analyzed by SPSS software, version 16 and $\mathrm{P}<0.05$ was accepted as statistically significant.

\section{Results}

\section{Clinical findings}

The observation showed no significant in food intake between different groups during the experiment. Most of the water consumption was observed in group A (an average of $27.7 \mathrm{~mL} / \mathrm{mouse} /$ day) in compared to group B $(21.9 \mathrm{~mL})$ and group C $(20.2 \mathrm{~mL})$. Semi liquid diarrhea was observed in the treated rats during the period of the experiment. All the rats subjected to the experiment showed an overall increase in body weights as compared to the initial weights. These results were as expected because the rats were in the growing stage. However, high dose treated rats showed lower average body weight. Although, body weights of animals increased in all groups, but there was no significant difference between groups (Figure 1).

Behavioral changes in both groups treated with $M$. officinalis were observed during the period, while the rats in the control group were normal and did not display significant changes in general behavior. Postural abnormalities, confusion, lethargy and reduced mobility were the main behavioral changes in the treated groups; however, they were more prominent in group B.

\section{Biochemical and hematological analyses}

The mean $\pm \mathrm{SE}$ of the hematological values in the treated and control rats are presented in Table 1. A significant decrease in the number of RBCs is observed in the treated rats in compared to the control group ( $\mathrm{P}=0.039)$. Clear leukocytosis was recorded in the treated rats with more than $4.2 \times 10^{3} / \mu \mathrm{L}$ WBCs in group $\mathrm{A}$ and $4.3 \times 10^{3} / \mu \mathrm{L}$ WBCs in group B, but these differences were not statistically significant in compared to the control group. The mean hemoglobin $(\mathrm{Hb})$ value was $13.78 \mathrm{dL} / \mathrm{gr}$ in the control group and a significant decreases were observed in group A (13.56 dL/gr) and $\mathrm{B}(12.26 \mathrm{dL} / \mathrm{gr}) \quad(\mathrm{P}=0.007)$. Other hematological parameters remained within physiological range.

The mean $\pm \mathrm{SE}$ of the biochemical values in the treated and control rats are presented in Table 2.

The ALT level was significantly lower in groups $\mathrm{A}$ and $\mathrm{B}$ when compared to the control group $(\mathrm{P}=.004)$. However, the
Serum AST was higher in rats treated with low dose of $M$. officinalis as compared to control and high dose treated rats, but these differences were not statistically significant $(\mathrm{P}=0.08)$. The highest levels of $\mathrm{CPK}$ and LDH were observed in group A (534 U/dL) and $\mathrm{B}(502 \mathrm{U} / \mathrm{dL})$, respectively, but there were no significant differences $(\mathrm{P}>0.05)$ as compared to the control group. Albumin concentrations decreased significantly in serum of treated rats in groups $B$ when compared to the group $\mathrm{A}$ and control $(\mathrm{P}=0.03)$.

Total protein concentrations in serum of rats dosed orally with $M$. officinalis extract decreased significantly in both treated groups $(\mathrm{P}=0.01)$. There were no significant differences in urea concentrations between all groups of rats. However, the creatinine concentrations were significantly higher $(\mathrm{P}<0.05)$ in rats treated with high dose of $M$. officinalis extract as compared to control and low dose treated rats. Oral administration of M. officinalis extract did not cause any significant changes in serum cholesterol and triglyceride.

\section{Histopathological changes}

No significant gross pathology was observed in any of the rats at necropsy. Livers and kidneys of all control rats were histologically normal, however these organs in the groups that received different doses of the extract showed mild, moderate, and severe tissue injuries.

The main histopathologic findings in the liver were included hepatocyte degeneration, congestion and dilation of sinusoids, bile ducts proliferation, infiltration of mononuclear cells around the portal area, polymorphism hepatocytes, central vein

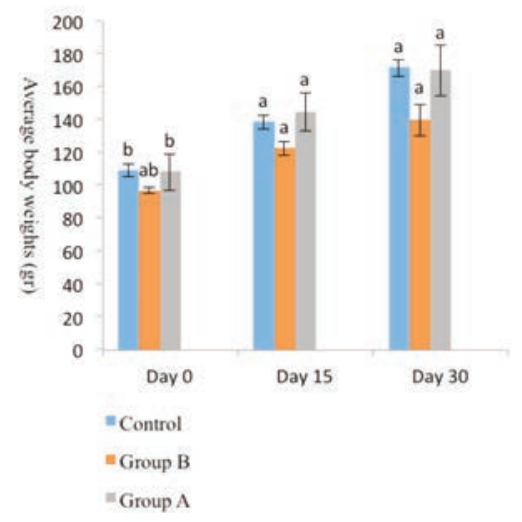

Figure 1. Effect of extract of $M$. officinalis on rats' body weight. Different letters in each column show significant differences between the groups $(P<0.05)$. Values are presented as mean \pm standard error. 
congestion and focal necrosis (Figure 2). The extent and severity of the lesions were dose dependent and they were more prominent in the group B, which received a high dose of extract (Table 3).

Histopathologic examination of the kidneys showed a tubular degeneration and necrosis, tubular and glomerular atrophy and congestion and diffused interstitial and glomerular hemorrhages (Figure 3). These lesions were significantly prominent in the group B. Histopathological changes scores of the study groups were shown in Table 4.

No pathological lesions were seen in the spleen, heart and lung in the rats of treated and control groups.

\section{Discussion}

Plants have been used for many thousands of years in food preservation, pharmaceuticals alternative medicine and natural therapies. ${ }^{9}$ Despite lack of scientific validation and toxicity evaluation, herbal medicines have received greater attention as an alternative to clinical therapy and the demand for these remedies has currently increased. 10

Herbs in their natural state vary in potency and may contain various bioactive substances, which can cause undesirable side effects. Therefore, it is necessary to investigate these plants scientifically to improve the quality of healthcare. The experimental screening method is important in order to ascertain the safety and efficacy of traditional and herbal products, and also to establish the active component of the herbal products. 11

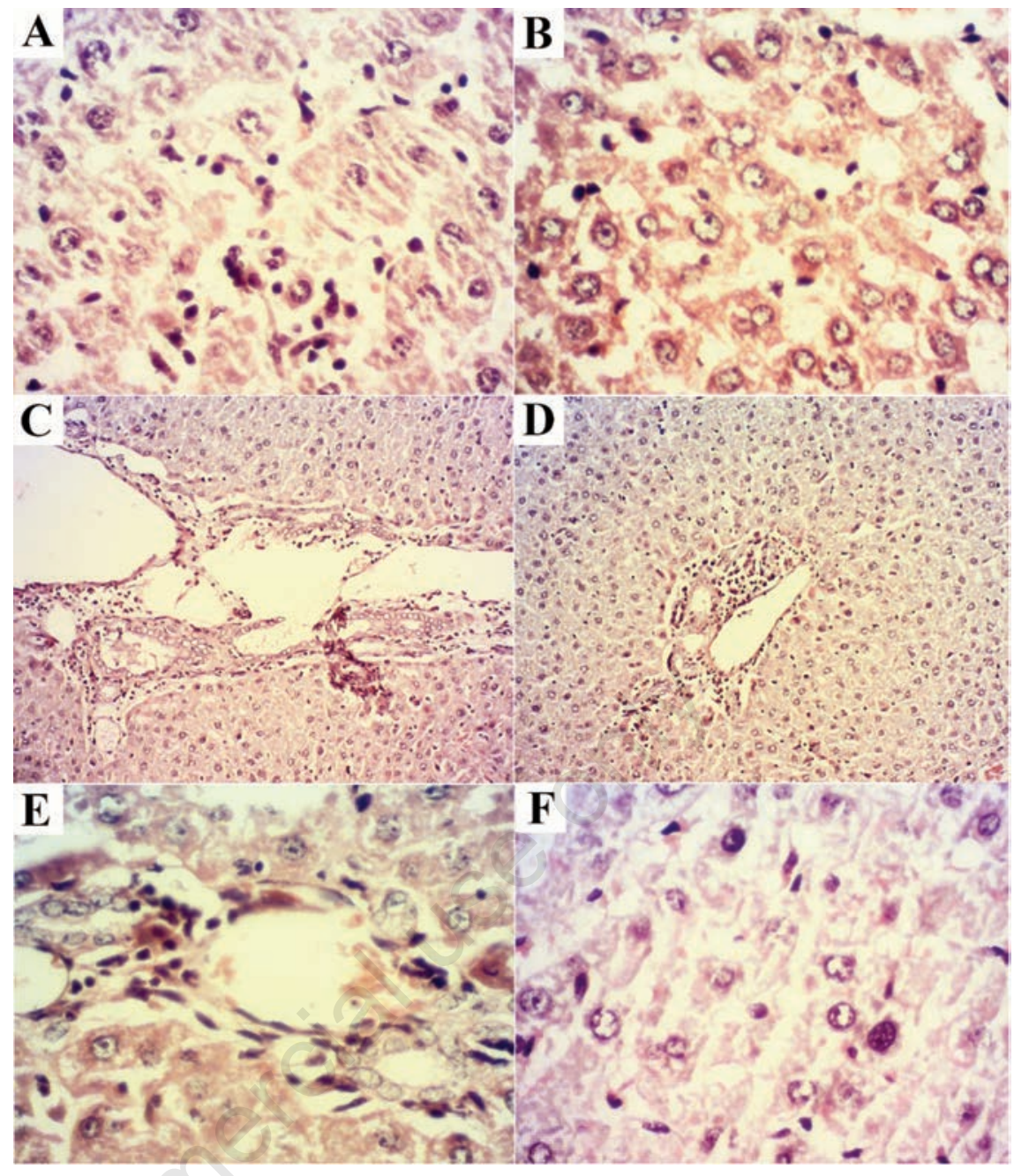

Figure 2. Liver sections of the treated groups. A) hepatocytes degeneration (H\&E; $\times 3000)$; B) congestion and dilation of sinusoids $(\mathrm{H} \& \mathrm{E} ; \times 3000)$; C) dilation of portal area and bile ducts proliferation $(\mathrm{H} \& \mathrm{E} ; \times 300) ; \mathrm{D})$ infiltration of mononuclear cells around the portal area $(\mathrm{H} \& \mathrm{E} ; \times 750) ; \mathrm{E})$ an increase of fibrous tissue around the portal area $(H \& E ; \times 3000)$; F) focal necrosis $(H \& E ; \times 3000)$.

Table 1. Hematological parameters in Sprague-Dawley rats after 30 days treatment with $M$. officinalis extract.

\begin{tabular}{|c|c|c|c|c|}
\hline Hematological parameters & Control & Low dose $(600 \mathrm{mg} / \mathrm{kg})$ & High dose (1200 mg/kg) & P value \\
\hline $\operatorname{RBC}(106 / \mu \mathrm{l})$ & $8.09 \pm 0.32$ & $7.77 \pm 0.32$ & $6.97 \pm 0.14$ & 0.039 \\
\hline WBC $(103 / \mu \mathrm{l})$ & $2.80 \pm 0.17$ & $4.20 \pm 0.04$ & $4.30 \pm 0.84$ & 0.087 \\
\hline HCT (\%) & $42.66 \pm 1.09$ & $47 \pm 2.61$ & $41.30 \pm 2.46$ & 0.194 \\
\hline PLT $(103 / \mu \mathrm{l})$ & $796.6 \pm 24.02$ & $1054.8 \pm 151.16$ & $855.4 \pm 163.11$ & 0.365 \\
\hline PCT (\%) & $0.70 \pm 0.06$ & $0.89 \pm 0.2$ & $0.75 \pm 0.22$ & 0.731 \\
\hline $\mathrm{MCV}(\mathrm{Fl})$ & $52.6 \pm 0.6$ & $60.4 \pm 0.92$ & $59 \pm 2.34$ & 0.007 \\
\hline $\mathrm{MCH}(\mathrm{pg})$ & $16.92 \pm 0.3$ & $17.5 \pm 0.81$ & $17.52 \pm 0.69$ & 0.761 \\
\hline MPV (Fl) & $8.64 \pm 0.6$ & $8.1 \pm 0.63$ & $8.22 \pm 0.83$ & 0.856 \\
\hline $\mathrm{MCHC}(\mathrm{g} / \mathrm{dL})$ & $31.9 \pm 0.23$ & $29.28 \pm 1.73$ & $30.26 \pm 2.08$ & 0.512 \\
\hline RDW (\%) & $15.34 \pm 0.11$ & $15.64 \pm 0.22$ & $14.52 \pm 0.56$ & 0.115 \\
\hline PDW (\%) & $5.66 \pm 0.23$ & $5.5 \pm 0.16$ & $7.18 \pm 0.76$ & 0.050 \\
\hline HGB (dL/gr) & $13.78 \pm 0.38$ & $13.56 \pm 0.13$ & $12.26 \pm 0.31$ & 0.007 \\
\hline
\end{tabular}

RBC, red blood cell count; WBC, white blood cell count; HTC, hematocrit; PLT, platelets; PCT, platelet hematocrit; MCV, mean corpuscular volume; MCH, mean corpuscular hemoglobin; MPV, mean platelets volume; MCHC, mean corpuscular hemoglobin concentration; RDW, red cell distribution width; PDW, platelet distribution width; HGB, hemoglobin. Values are expressed as mean \pm standard error. P<0.05 was considered significant. 
Chronic toxicity studies in animals are valuable in predicting potential toxic effects of a substance or a plant extract from which the response may be correlated with human. It also gives an idea about the organ system involvement. 9

M. officinalis is a medicinal plant and it is widely used in Iran as well as western Asia and northern Africa. Although, some recent studies on Melissa have shown its therapeutic effects and chemical composition, but there is no information about the clinical effects of long-term administration of M. officinalis and its potential toxicological effects on the various organs.

In the present study, the evaluation reports showed an overall increase in body weights of the rats in all groups as compared to the initial weights. These results were as expected because the rats were in the growing stage. However, higher dose treated rats showed lower average body weight. This may be due to the presence of some substances in M. officinalis that could have limited the food intake.
In this experiment, there is no significant change in hematological parameters like WBC, HCT, PLT, PCT, MCH, MPV, MCHC, RDW and PDW in the treated animals. The red blood cell was found to be significantly decreased $(\mathrm{P}<0.05)$ in Groups $\mathrm{A}$ and $\mathrm{B}$. Additionally, clear leukocytosis was recorded in the treated rats, but these differences were not statistically significant in compared to the control group.

The hematopoietic system is one of the most sensitive targets for toxic chemicals and an important index of physiological and pathological status in human and animal.12

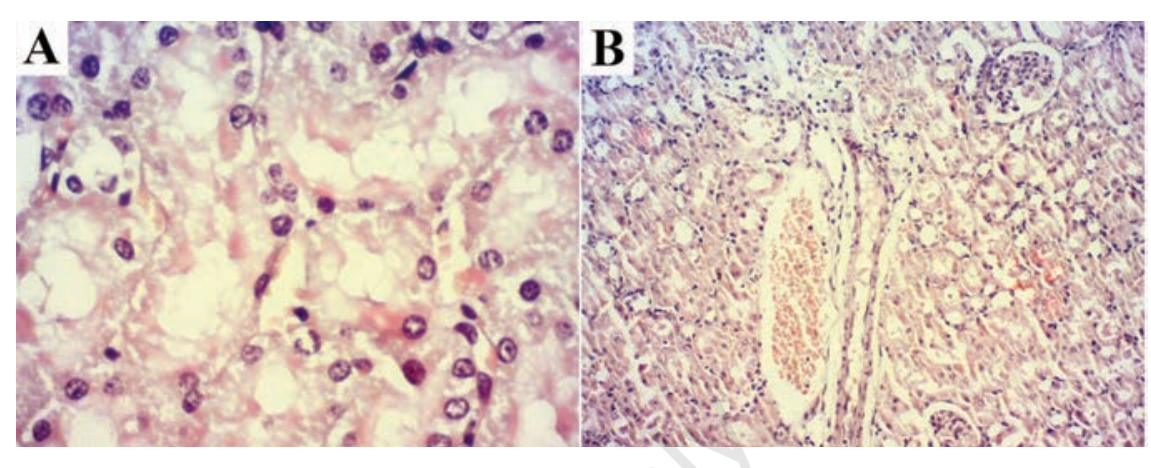

Figure 3. Kidney sections of the treated groups. A) tubular degeneration and necrosis $(\mathrm{H} \& \mathrm{E} ; \times 3000)$; B) tubular and glomerular atrophy and congestion and diffused interstitial and glomerular hemorrhages $(\mathrm{H} \& \mathrm{E} ; \times 750)$.

Table 2. Biochemical parameters in Sprague-Dawley rats after 30 days treatment with $M$. officinalis extract.

\begin{tabular}{lcccc}
\hline Biochemical parameters & Control & Low dose $(600 \mathrm{mg} / \mathrm{kg})$ & High dose $(1200 \mathrm{mg} / \mathrm{kg})$ & P value \\
ALT (U/dL) & $98.4 \pm 1.8$ & $126 \pm 3.58$ & $134 \pm 9.98$ & 0.004 \\
AST (U/dL) & $49 \pm 4.50$ & $53 \pm 1.92$ & $49 \pm 2.9$ & 0.620 \\
\hline LDH (U/dL) & $488.50 \pm 18.34$ & $428.66 \pm 22.40$ & $502 \pm 25.6$ & 0.330 \\
CPK (U/dL) & $482 \pm 22.9$ & $534 \pm 34.7$ & $446 \pm 19.1$ & 0.240 \\
\hline ALB (mg/dL) & $4.06 \pm 0.05$ & $3.94 \pm 0.08$ & $3.46 \pm 0.19$ & 0.030 \\
TG (mg/dL) & $46.40 \pm 0.67$ & $46 \pm 0.7$ & $45.40 \pm 5.16$ & 1.00 \\
\hline Cholesterol (mg/dL) & $83 \pm 0.89$ & $89.2 \pm 2.69$ & $83.2 \pm 9$ & 0.890 \\
TP (mg/dL) & $6.80 \pm 0.08$ & $5.80 \pm 0.08$ & $5.90 \pm 0.05$ & 0.010 \\
\hline Creatinine (g/dL) & $0.55 \pm 0.05$ & $0.60 \pm 0.07$ & $0.68 \pm 0.04$ & 0.008 \\
Urea (mg/dL) & $56.2 \pm 3.83$ & $61.6 \pm 6.06$ & $60.6 \pm 7.43$ & 0.805 \\
\hline
\end{tabular}

ALT, anine-aminotransferase; AST, aspartate-aminotransferase; LDH, lactate dehydrogenase; CPK, creatine phosphokinase; ALB, albumin; TG, thrombin generation; TP, thrombin potential. Values are expressed as mean \pm standard error. $\mathrm{P}<0.05$ was considered significant.

Table 3. Histopathological change scores of liver in all study groups.

\begin{tabular}{|c|c|c|c|c|}
\hline Histopathological changes & Control & Low dose (600 mg/kg) & High dose (1200 mg/kg) & P value \\
\hline Hepatocyte degeneration & - & $2+$ & $2+$ & 0.001 \\
\hline Congestion of sinusoids & - & + & $2+$ & 0.003 \\
\hline Dilation of sinusoids & - & + & $2+$ & 0.001 \\
\hline Congestion of portal vein & + & + & + & 0.003 \\
\hline Dilation of portal area & - & + & $3+$ & 0 \\
\hline Central vein congestion & - & $2+$ & $2+$ & 0.001 \\
\hline Bile ducts proliferation & - & - & + & 0 \\
\hline Portal area inflammation & + & $2+$ & $2+$ & 0 \\
\hline Polymorphism hepatocytes & - & + & $2+$ & 0 \\
\hline Prominent nucleolus & $2+$ & $2+$ & $2+$ & 1 \\
\hline Hepatocytes with eosinophilic cytoplasm & + & $2+$ & $2+$ & 0.005 \\
\hline Focal necrosis & - & + & $2+$ & 0 \\
\hline Portal area fibrosis & - & + & + & 0.005 \\
\hline
\end{tabular}

$\mathrm{P}<0.05$ was considered significant. No lesion (-); mild (+); moderate (2+); severe (3+). 
Table 4. Histopathological change scores of kidney in all study groups.

\begin{tabular}{|c|c|c|c|c|}
\hline Histopathological changes & Control & Low dose $(600 \mathrm{mg} / \mathrm{kg})$ & High dose (1200 mg/kg) & P value \\
\hline Tubular necrosis & - & + & $3+$ & 0 \\
\hline Glomerular atrophy & - & - & + & 0.001 \\
\hline Tubular atrophy & - & + & $2+$ & 0.001 \\
\hline Intratubular protein casts & - & - & + & 0.011 \\
\hline Mononuclear cell infiltration & - & - & - & 1 \\
\hline Interstitial fibrosis & - & - & - & 1 \\
\hline Glomerular congestion & + & + & $2+$ & 0 \\
\hline Interstitial tissue congestion & + & $2+$ & $2+$ & 0.004 \\
\hline
\end{tabular}

$\mathrm{P}<0.05$ was considered significant. No lesion (-); mild (+); moderate (2+); severe (3+).

Hematological changes such as anemia are often accompanied with bone marrow toxicity. ${ }^{13}$ Additionally, lysis of blood cells due to administration of some agents can be another reason for anemia. ${ }^{9}$

In this study, although the number of RBC decreased after administration of $M$. officinalis in compared to control, but their number remained in the normal range and suggesting that there is no lysis of blood cells and the decrease in RBC count may be attributed to the less production or release of $\mathrm{RBC}$ in the bone marrow. Increase in WBC may indicate the impact of $M$. officinalis in stimulating the immune system of the treated groups.

Transaminases, LDH, and alkaline phosphatases are good indices of liver, heart and kidney damage respectively. An increase in the level of AST and ALT in the blood reflects the structural and functional dysfunction of hepatocellular membrane or cell rupture, and thereby indicate liver damage associated with tissue injury and the reflection of hepatic toxicity. ${ }^{1}$

In the present study, the ALT level was significantly higher in the treated rats when compared to the control. However, AST level was not affected by $M$. officinalis, when is compared treated and controls animals. The significant increase in the level of ALT can be due to liver damage that is consistent with the histopathological findings. Although it was expected that the treated rats showed an increase in AST level, the lack of significant alterations in the AST level is not reasonable. However, some references have mentioned that the ALT level is the hallmark of hepatocyte injuries in the rat, not AST and the increase in ALT without alterations in the AST level is logical. ${ }^{14}$

There were no significant differences in the LDH and CPK levels between treated and control groups. This indicates that the serum levels of these parameters were not affected by the consumption of $M$. officinalis.

There were no significant changes found in the cholesterol and triglyceride concentrations in serum of treated groups as compared to control. The liver is the site of cholesterol disposal or degradation and the major site of synthesis. ${ }^{10}$ Since, no significant changes in cholesterol levels in this study, it suggests that $M$. officinalis extract had no effects on the cholesterol metabolism of the treated rats.

Renal glomerular functions are assessable by measuring the plasma creatinine and urea concentrations. Urea is synthesized in the liver as the primary by-product of deamination of amino acids. Creatinine is a by-product from muscle as its concentration in blood will be affected by any changes in muscle mass. ${ }^{15,16}$ Hence, increase in plasma creatinine or urea concentration would indicate renal damages.

In this experiment, the treated rats showed a significant increase in the creatinine concentration in compared to the control group. This indicates some injuries to the kidneys especially in the glomerular renal functions as a result of long term $M$. officinalis extract administration. There were no significant differences in urea concentrations between all groups of rats.

Total protein and albumin concentrations decreased significantly in the treated rats when compared to the control. Generally, diarrhea is assumed to cause intestinal protein loss and consequently a reduced total protein serum concentration. ${ }^{17}$

Histological examination is the golden standard for evaluating pathological changes in tissues and organs when exposed to the foreign compounds which may or not to be toxic. 10

The main histopathologic lesions in the liver due to administration of $M$. officinalis were included hepatocyte degeneration, congestion and dilation of sinusoids, proliferation of bile ducts, polymorphism hepatocytes and infiltration of mononuclear cells around the portal area. The lesions varied from mild to moderate. These pathological lesions accompany with an increase in the
ALT level indicate a mild to moderate liver injury following long-term consumption of M. officinalis extract.

Histological analysis of kidney showed a mild to moderate tubular degeneration and necrosis, tubular and glomerular atrophy and congestion in the treated rats. These findings are consistent with an increase in plasma creatinine concentration in the treated animals.

Histopathological examination of other organs from treated and control animals showed normal structures, suggesting no pathological changes and morphological disturbances caused due to the administration of M. officinalis extract.

\section{Conclusions}

According to the biochemical, hematological and histopathological analysis, $M$. officinalis was found to be toxic when longterm oral administration was performed in rats. Alterations in some hematological and biochemical parameters and also mild to moderate lesions in the liver and kidney support the fact that they are plausible target organs for adverse effects of $M$. officinalis.

This study showed that the use of extract of $M$. officinalis is not completely safe and much further studies should be performed to verify the safety of this plant in the traditional medicine.

The findings suggest that long-term administration of $M$. officinalis extract even at low doses induces hepatic and renal lesions in rats.

\section{References}

1. Morales G, Paredes A, Olivares A, et al. Acute oral toxicity and anti-inflammatory activity of hydroalcoholic extract from Lampaya medicinalis Phil in rats. 
Biol Res 2014;47:6-13.

2. George P. Concerns regarding the safety and toxicity of medicinal plants: an overview. J App Pharm Sci 2011;1:404.

3. Harizal SN, Mansor SM, Hasnan J, et al. Acute toxicity study of the standardized methanolic extract of Mitragyna speciosa Korth in Rodent. J Ethnopharmacol 2010;131:404-9.

4. Herodez SS, Hadolin M, Skerget M, et al. Analytical, Nutritional and Clinical Methods Solvent extraction study of antioxidants from Balm (Melissa officinalis L.) leaves Food Chem 2003;80:275-82.

5. Aharizad S. Study of genetic diversity in lemon balm (Melissa officinalis 1.) populations based on morphological traits and essential oils content. Ann Biol Res 2012;3:5748-53.

6. Blumenthal M, Goldberg A, Brinckmann J. Herbal medicine. Expanded Commission E Mongraphs. Newton, MA: Integrative Medicine Communications 2000;123:230-2.

7. Moradkhani H, Sargsyan E, Bibak H, et al. A valuable medicine plant: A review. J Med Plants Res 20104;25:2753-9.

8. Ozden H, Bildirici K, Ustuner D, et al. Histopathologic examination of rat liver after experimental application of fluoxetine. Turk Journal Ecopathol 2005;11:9-15.

9. Uma M, Suresh M, Thulasiraman K, et al. Chronic toxicity studies of aqueous leaf extract of Indian traditional medicinal plant Ocimum tenuiflorum (Linn.) in rats. Eur J Exp Biol 2013;3:240-7.

10. Pillai PG, Suresh P, Mishra G, et al. Evaluation of the acute and subacute toxicity of the methanolic leaf extract of Plectranthus amboinicus (Lour) Spreng in Balb C mice. European J Exp Biol 2011;1:236-45.

11. Mythilpriya R, Shanthi P, Sachdanandam P. Oral acute and subacute toxicity studies with Kalpaamurthaa, a modified in digenous prepration on rats. J Health Sci 2007;53:351-8.

12. Li X, Luo Y, Wang L, et al. Acute and subacute toxicity of ethanol extracts from Salvia przewalskii Maxim in rodents. J Ethnopharmacol 2010;131:110-5.

13. Rhiouani H, El-Hilalya J, Israili ZH, et al. Acute and subchronic toxicity of an aqueous extract of the leaves of Herniaria glabra in rodents. J Ethnopharmacol 2008;118:378-86.

14. Boone L, Meyer D, Cusick P, et al. Selection and interpretation of clinical pathology indicators of hepatic injury in preclinical studies Vet Clin Pathol 2005;34:182-8

15. Moshi MJ, Lutale JJK, Rimoy GH, et al. The effect of Phyllanthus amarus aqueous extract on blood glucose in non-insulin dependent diabetic patients. Phytother Res 2001;15:577-80.

16. Chua HP, Murugaiyah M, Rohani MY, et al. Toxicological evaluation of dried kacangma (Leonurus sibiricus) in rats: I. Blood chemistry, body and organ weight changes. J Trop Agric and Fd Sc 2006;34:57-65.

17. Latimer KS, Mahaffey EA, Prasse KW. Veterinary laboratory medicine: clinical pathology. 4th ed. Iowa City: Iowa State Press; 2003. 